\title{
Análisis de efectividad de la ventilación de alta frecuencia oscilatoria en pacientes pediátricos con insuficiencia respiratoria aguda en un centro de alta complejidad Effectiveness analysis of high-frequency oscillatory ventilation in pediatrics patients with acute respiratory failure in a tertiary hospital
}

\author{
Dr. Pedro Taffarel ${ }^{a}$, Dr. Germán Bonetto ${ }^{a}$, Dr. Facundo Jorro Barón ${ }^{a}$, \\ Dr. Jorge Selandari ${ }^{a}$ y Dr. Jorge Sasbón ${ }^{a}$
}

\section{RESUMEN}

Introducción. La ventilación de alta frecuencia oscilatoria (VAFO) es un recurso terapéutico ante la hipoxemia refractaria a la asistencia respiratoria mecánica convencional (ARMc), y en el tratamiento del síndrome de escape aéreo. Un metaanálisis reciente concluyó que la VAFO parece disminuir la mortalidad en niños posneonatales y adultos. En este contexto, es importante la evaluación de los resultados en el uso rutinario de esta tecnología.

Objetivos. Principal: Analizar la efectividad de la VAFO en la práctica rutinaria en un centro que no dispone de oxigenación por membrana de circulación extracorpórea.

Secundarios: Describir los datos demográficosy las causas de insuficiencia respiratoria grave de los pacientes que requirieron VAFO. Examinar la relación entre factores predictores potenciales y la ocurrencia de mortalidad.

Población y métodos. Estudio retrospectivo en el cual se analizaron las historias clínicas de los pacientes que requirieron VAFO en las Unidades de Cuidados Intensivos de un hospital pediátrico terciario en el período 01/01/2008 - 01/07/2010.

Resultados. Se analizaron 76 ingresos a VAFO en 69 pacientes. El 80\% de tales ingresos tenían diagnóstico de infección respiratoria aguda baja o sepsis. El 62,3\% (n=43) de los casos presentaban enfermedad crónica subyacente. La mayoría de los ingresos fueron por hipoxemia refracta-

a. Hospital de Pediatría SAMIC "Prof. Dr. Juan P. Garrahan". Ciudad Autónoma de Buenos Aires.

Correspondencia:

Dr. Jorge Selandari. jselandari@intramed.net

Conflicto de intereses: Ninguno que declarar.

Recibido: 19-10-2011 Aceptado: 3-4-2012 ria $(93,4 \%)$. Se halló que los pacientes fallecidos presentaban peores condiciones clínicas al ingreso, mayor compromiso multiorgánico, peor oxigenación y compromiso pulmonar.

Conclusión. En una población con alta prevalencia de enfermos crónicos, oncológicos e inmunodeprimidos, el uso rutinario de la VAFO se asoció con un $33,4 \%$ de supervivencia. Se necesitan más estudios pronósticos que permitan una selección más eficaz de los pacientes candidatos a esta tecnología.

Palabras clave: ventilación de alta frecuencia, sindrome de dificultad respiratoria aguda (no adulto), lesión pulmonar inducida por ventilación mecánica, pediatría.

\section{SUMMARY}

Introduction. High frequency oscillatory ventilation (HFOV) is a rescue therapy for hypoxemic patients who deteriorate in conventional mechanical ventilation and/or for the air-leak syndrome treatment. A recent meta analysis showed that HFOV might have reduced mortality in pediatric and adult patients compared with conventional ventilation. In this context it's important to evaluate the effectiveness of this method in everyday use.

Objectives. Main: To analize the effectiveness of HFOV in everyday practice in a center without extra corporeal membrane oxygenation (ECMO) capabilities.

Secondary: To describe demographics and causes of severe respiratory failure of patients requiring HFOV. To assess the relationship between potential predictors and the occurrence of mortality.

Population and methods. Retrospective study analyzing medical records of all patients that required HFOV in a tertiary care pediatric hospital pediatric intensive care units between march $1^{\text {st }} 2008$ and july $1^{\text {st }} 2010$.

Results. Sixty-nine patients received 76 HFOV treatments. Eighty percent were diagnosed with acute lower respiratory infection or sepsis and $62.3 \%(n=43)$ had preexisting chronic co-morbidities. The majority of HFOV treatments were because refractory hypoxemia (93.4\%).

Non survivors patients had worse clinical status at PICU admission, higher multiorgan failure, worse oxygenation and pulmonary condition. Conclusions. Everyday use of HFOV in a population with high incidence of chronic, oncologic and/or immunocompromised patients was associated with a survival of $33.4 \%$. More prognostic studies are needed for a more effective selection of HFOV candidates.

Keywords: high-frequency ventilation, respiratory distress syndrome, no adult, ventilator-induced lung injury, pediatrics.

http:/ /dx.doi.org/10.5546/aap.2012.214 


\section{INTRODUCCIÓN}

El síndrome de dificultad respiratoria aguda (SDRA) es un cuadro clínico secundario a una lesión pulmonar o extrapulmonar que resulta en un daño a la membrana alvéolo-capilar, con el consiguiente aumento de su permeabilidad y edema pulmonar no cardiogénico. Se trata de una enfermedad parenquimatosa restrictiva, donde la distensibilidad pulmonar está reducida y los volúmenes pulmonares gravemente disminuidos, especialmente a expensas de la capacidad residual funcional; su principal característica clínica es la hipoxemia relativamente refractaria al aumento de la fracción inspirada de oxígeno $\left(\mathrm{FIO}_{2}\right) \cdot{ }^{1-4}$

En la actualidad se acepta que, aunque la asistencia respiratoria mecánica (ARM) es muchas veces indispensable para la supervivencia del paciente, también es parte del problema del SDRA, ya que datos experimentales y clínicos demuestran que la ventilación mecánica es capaz de generar una lesión histopatológicamente y clínicamente indiferenciable del SDRA..$^{5-8}$

El clásico estudio de Webb y cols., ${ }^{9}$ fue el primero en demostrar, inequívocamente, que la ventilación mecánica puede producir edema pulmonar; y al mismo tiempo, fue una demostración de que la presión positiva al fin de la espiración (PEEP), con una estrategia de limitación del volumen corriente, protege de la lesión pulmonar asociada a la ventilación mecánica. En esta línea de conocimiento, hallazgos como los de Dreyfuss y cols. ${ }^{10}$ en estudios experimentales a finales de los ochenta, sobre la relación entre el empleo de alto volumen corriente y el incremento de la lesión pulmonar; los estudios hechos por el grupo de Gattinoni mediante tomografía axial computarizada y el desarrollo del concepto de baby-lung, ${ }^{11}$ que muestran que, en realidad, en el SDRA sólo es una pequeña proporción del pulmón la que interviene en el intercambio gaseoso y el resto del órgano se encuentra colapsado o inundado y, por último, los resultados clínicos del estudio de la ARDS Network, ${ }^{12}$ que confirmó la necesidad de emplear bajo volumen corriente en estos pacientes, fueron sin duda avances importantes en el conocimiento de esta entidad y han influido favorablemente en su pronóstico.

La ventilación oscilatoria de alta frecuencia (VAFO) es un modo de ARM que en estudios en animales demuestra lograr los objetivos fisiopatológicos de protección pulmonar. Se caracteriza por el uso de frecuencias suprafisiológicas y vo- lúmenes corrientes cercano el espacio muerto, y de esta manera puede otorgar presiones medias en la vía aérea (Paw) más altas que la ventilación convencional, pero con menores presiones pico y menores oscilaciones de volumen con, por ende, menor volutrauma y barotrauma, y al permitir descender la $\mathrm{FIO}_{2}$, menor toxicidad de $\mathrm{O}_{2} \cdot{ }^{13,14} \mathrm{De}$ esta manera la VAFO es un recurso terapéutico ante la hipoxemia refractaria a la ARMc, y en el tratamiento del síndrome de escape aéreo. En el único estudio aleatorizado pediátrico posneonatal, la VAFO mostró en los pacientes tratados un requerimiento menor de oxigenoterapia a los 30 días. ${ }^{15}$ Un metaanálisis reciente concluyó que la VAFO parece disminuir la mortalidad en niños posneonatales y adultos. ${ }^{16}$

\section{OBJETIVOS}

Principal:

- Analizar la efectividad de la VAFO en la práctica rutinaria en un centro terciario que no dispone de oxigenación de membrana extracorpórea (ECMO), en el período enero 2008 julio 2010.

Secundarios:

- Describir los datos demográficos y las causas de insuficiencia respiratoria grave de los pacientes que requirieron VAFO.

- Examinar la relación entre factores predictores potenciales y la ocurrencia de mortalidad.

\section{POBLACIÓN Y MÉTODOS}

Se realizó un estudio retrospectivo en el cual se analizaron las historias clínicas de los pacientes que requirieron VAFO en las unidades de Cuidados Intensivos Polivalentes del Hospital de Pediatría "Prof. Dr. Juan P. Garrahan" en el período 01/01/2008-01/07/2010. Las historias clínicas fueron revisadas por alguno de los dos primeros autores, resolviéndose las dudas por consenso entre ambos. Se utilizó, en el período 2008, el oscilador SensorMedics 3100A y, a partir del año 2009, se incorporó como opción el respirador SLE 5000. Se revisó edad, diagnóstico de base, PIM2 (índice de mortalidad pediátrica 2), causa de SDRA (categorizado como primario o secundario si el factor desencadenante era pulmonar o extrapulmonar, respectivamente), puntaje de disfunción multiorgánica (PELOD: Pediatric Logistic Organ Dysfunction), ${ }_{17}$ Paw, índice de oxigenación (IO), puntaje de Murray de compromiso pulmonar ${ }^{18}$ y PAFI $\left(\mathrm{PaO}_{2} / \mathrm{FIO}_{2}\right)$ al 
ingreso a VAFO, días de ventilación mecánica convencional (VMC) previa al ingreso a VAFO y su duración (días), presencia de escape de aire al ingreso a VAFO (evidencia radiológica de enfisema intersticial, neumotórax, neumomediastino, etc. o enfisema subcutáneo consignado en la historia clínica), Paw de salida de VAFO, óbito en UCIP y su causa. Se agregó, en el período 01/01/2009-01/07/2010, el análisis de las siguientes variables: $\mathrm{PCO}_{2}$ (presión arterial de dióxido de carbono) al ingreso a VAFO, hipotensión (caída del 20\% de la tensión arterial media o requerimiento de fármacos vasoactivos) a partir del ingreso a VAFO, necesidad de adrenalina $>0,25 \mu \mathrm{g} / \mathrm{kg} / \mathrm{min}$, como expresión de bajo gasto cardíaco o hemofiltración como terapia de reemplazo renal, Paw máxima en alta frecuencia y necesidad de utilizar $\mathrm{FIO}_{2}>0,6$ por más de 24 $h$ mientras se encontraba en VAFO.

$\mathrm{El}$ ingreso a VAFO en nuestro servicio se considera en pacientes que requieren $\mathrm{FIO}_{2}>0,7$, PEEP $>15-20$ para lograr una $\mathrm{SatO}_{2} 85-90 \%$ con presiones pico $>35-40 \mathrm{cmH}_{2} \mathrm{O}$ (en modo presión control), de modo de mantener un $\mathrm{pH}>7,25$ y después del fracaso del decúbito prono; otra indicación aceptada es el tratamiento o la prevención de los síndromes de escape de aire. ${ }^{14}$ No se emplean maniobras de reclutamiento ni óxido nítrico en forma rutinaria en el tratamiento de la hipoxemia refractaria de estos pacientes. La indicación final es decidida por el intensivista a cargo del enfermo. Una vez en VAFO, el paciente es tratado según lineamientos publicados. ${ }^{14}$

\section{Análisis estadístico}

Se realizó con el programa STATA 9.0 para Windows ${ }^{\circledR}$ (StataCorp, College Station, Texas, EE.UU.). De acuerdo a la distribución de las variables, normal o no-normal, sus valores fueron expresados en medias y desvíos estándar o medianas e intervalo intercuartilo y analizadas con la prueba de la T o de la suma ordinal de Wilcoxon, respectivamente. Se utilizó el estadístico de $\chi^{2}$ para comparar las variables dicotómicas (Pearson o Fisher de acuerdo al número de observaciones en cada grupo). Una p menor de 0,05 se consideró significativa.

\section{RESULTADOS}

En el período 01/01/2008-01/07/2010 se hallaron 85 ingresos a VAFO. Nueve se excluyeron del estudio por ausencia de datos de oxigenación
TABLA 1. Principales características de los 76 ingresos a VAFO

\begin{tabular}{|c|c|}
\hline Variable & $n(\%)-X \pm D E-M e / R I Q$ \\
\hline \multicolumn{2}{|l|}{ Casos ingresados por año } \\
\hline-2008 & $25(33 \%)$ \\
\hline-2009 & $32(42 \%)$ \\
\hline - 2010 (primer semestre) & $19(25 \%)$ \\
\hline Edad (meses) & $12,5 / 5-54$ \\
\hline Peso corporal (kg) & $10,5 / 6-16$ \\
\hline PIM2 (\%) & $15,2 \pm 19,75$ \\
\hline \multicolumn{2}{|l|}{ Categorías diagnósticas } \\
\hline - Infección respiratoria aguda baja & $45(59 \%)$ \\
\hline - Sepsis & $16(21 \%)$ \\
\hline - Traumatismos & $5(7 \%)$ \\
\hline - Otros & $10(13 \%)$ \\
\hline Enfermedad crónica subyacente & $43(62,3 \%)$ \\
\hline \multicolumn{2}{|l|}{ Indicación de ingreso a VAFO } \\
\hline 1. Hipoxemia refractaria (SDRA) & $71(93,4 \%)$ \\
\hline - SDRA primario & $49(64,5 \%)$ \\
\hline - SDRA secundario & $22(28,9 \%)$ \\
\hline - Escape aéreo concomitante & $5(7 \%)$ \\
\hline 2. Escape aéreo & $3(4,2 \%)$ \\
\hline 3. Hipercapnia refractaria & $2(2,6 \%)$ \\
\hline VMC pre-VAFO (días) & $8,63 \pm 16,2$ \\
\hline \multicolumn{2}{|l|}{ Equipo utilizado } \\
\hline SensorMedics $3100^{a}$ & $52(68,4 \%)$ \\
\hline SLE 5000 & $24(31,6 \%)$ \\
\hline \multicolumn{2}{|l|}{ Variables al ingreso a VAFO } \\
\hline Paw (mmHg) & $21,2 \pm 5,3$ \\
\hline $\mathrm{IO}$ & $25,6 \pm 13$ \\
\hline PAFI (mmHg) & $98,47 \pm 53,38$ \\
\hline Puntaje de Murray & $3 \pm 0,66$ \\
\hline $\mathrm{PCO}_{2}(\mathrm{mmHg})$ & $60,27 \pm 18,43$ \\
\hline PELOD al ingreso a VAFO (\%) & $15,37 \pm 19,3$ \\
\hline Duración media de VAFO (días) & $7,22 \pm 6,94$ \\
\hline \multicolumn{2}{|l|}{ Oxigenoterapia a los 30 días de VAFO } \\
\hline No & $11(15,9 \%)$ \\
\hline Sí & $16(23,18 \%)$ \\
\hline Fallecidos & $42(60,8 \%)$ \\
\hline Mortalidad & $46(66,6 \%)$ \\
\hline - Falla multiorgánica & $52,2 \%$ \\
\hline - Hipoxemia refractaria & $41,3 \%$ \\
\hline - Muerte cerebral & $6,5 \%$ \\
\hline \multirow{2}{*}{\multicolumn{2}{|c|}{$\begin{array}{l}\text { Datos adicionales registrados en el } \\
\text { período 01/01/2009 - 01/07/2010 } \\
\text { (51 ingresos }[67 \%] \text { ) }\end{array}$}} \\
\hline & \\
\hline Hipotensión arterial & $18(35,3 \%)$ \\
\hline \multicolumn{2}{|l|}{ Fármacos vasoactivos desde } \\
\hline el ingreso a VAFO & $9(17,6 \%)$ \\
\hline $\mathrm{FIO}_{2}>0,6$ por más de $24 \mathrm{~h}$ & $38(74,5 \%)$ \\
\hline Adrenalina $>0,25 \mu \mathrm{g} / \mathrm{kg} / \mathrm{min}$ & $33(64,7 \%)$ \\
\hline Hemofiltración & $8(15,68 \%)$ \\
\hline
\end{tabular}

n (\%): número (\%); X \pm DE: media \pm desvío estándar; Me/RIQ: mediana e intervalo intercuartilo.

PIM2: índice de mortalidad pediátrica; VMC: ventilación mecánica convencional; VAFO: ventilación oscilatoria de alta frecuencia; SDRA: síndrome de dificultad respiratoria aguda; Paw: presión media de la vía aérea; IO: índice de oxigenación; PAFI:PaO $/ \mathrm{FIO}_{2} ;$ PELOD:puntaje de disfunción multiorgánica (Pediatric Logistic Organ Dysfunction). 
y ventilación mecánica en la historia clínica. Se analizaron entonces 76 ingresos a VAFO en 69 pacientes cuyas características principales se muestran en la Tabla 1. El 80\% de los ingresos tuvieron como diagnóstico principal infección respiratoria aguda baja o sepsis. De los 43 casos (62,3\%) que presentaban enfermedad subyacente, $19(44,18 \%)$ eran oncólogicos y 7 eran inmunocomprometidos de causa no oncológica ( 2 inmunodeficiencia combinada grave, 2 sida, 1 lupus eritematoso sistémico y una hepatitis autoinmunitaria). Los pacientes con enfermedad subyacente tuvieron una mayor probabilidad de fallecer (OR 3,6 IC95\% 1,4-9,2). La indicación de ingreso a VAFO se clasificó como: 1) Hipoxemia refractaria $\left(\mathrm{FIO}_{2}>0,7, \mathrm{PEEP}>15-20\right.$ para lograr una $\left.\mathrm{SatO}_{2} 85-90 \%\right)$, o 2) Escape aéreo o 3 ) Hipercapnia refractaria $(\mathrm{pH}<7,25$ con $\mathrm{PaCO} 2>$ 45 en ausencia de hipoxemia refractaria y/o escape aéreo). La mayoría de los ingresos fueron por hipoxemia refractaria y en el total de la muestra hubo 8 casos $(10,5 \%)$ que al ingresar a VAFO presentaban escape aéreo, 5 en forma concomitante con la hipoxemia que motivó el ingreso y 3 como indicación primaria. Estos últimos tenían como diagnóstico de base insuficiencia hepática aguda por hepatitis autoinmunitaria, leucemia linfoidea aguda y atrofia espinal de tipo I y los 3 fallecieron (Tabla 1).
A fin de determinar la existencia de factores predictores de mortalidad, se compararon los ingresos a VAFO de acuerdo a que hubiesen sobrevivido o no (Tabla 2).

En el análisis de las variables, se halló que los pacientes fallecidos presentaban peores condiciones clínicas al ingreso a UCIP, reflejado por mayor prevalencia de enfermedades crónicas subyacentes y mayor puntaje de PIM2. Al ingreso a alta frecuencia, los pacientes fallecidos mostraron mayor compromiso multiorgánico en el puntaje de PELOD, peor oxigenación (PAFI e IO) y compromiso pulmonar evaluado por el puntaje de Murray. De la misma manera, la mala evolución en VAFO fue altamente predictora de desenlace fatal, como se ve en la mayor proporción de necesidad $\mathrm{FIO}_{2}>0,6$ por más de 24 h, valores máximos de Paw, mayor requerimiento de adrenalina a altas dosis y mayor requerimiento de terapias de reemplazo renal en los pacientes fallecidos. Por último, aunque no fue significativa, se registró una tendencia a mayor cantidad de días de ARMc previo a la utilización de VAFO en los pacientes no sobrevivientes $(10,3$ contra 6,1 días $\mathrm{p}=0,19$ ).

Se analizó, comparativamente, los pacientes que recibieron VAFO con el oscilador SensorMedics 3100A contra el SLE5000, a fin de deter-

TABLA 2. Comparación de sobrevivientes y no sobrevivientes

\begin{tabular}{|c|c|c|c|}
\hline Variables & Sobrevivientes (30 ingresos) & No sobrevivientes (46 ingresos) & $\mathbf{P}$ \\
\hline Edad (meses) & $35 \pm 46,7$ & $42,98 \pm 53,43$ & 0,51 \\
\hline Enfermedad crónica subyacente $(n=43)$ & $28 \%(n=12)$ & $72 \%(n=31)$ & 0,01 \\
\hline PIM2 (\%) & $7,83 \pm 12,4$ & $20 \pm 22$ & 0,0017 \\
\hline Paw al ingreso a VAFO (mmHg) & $19,85 \pm 5,17$ & $22 \pm 5,27$ & 0,071 \\
\hline IO al ingreso a VAFO & $21,19 \pm 9$ & $28,62 \pm 14,4$ & 0,014 \\
\hline PAFI & $113 \pm 70$ & $88,9 \pm 36,69$ & 0,023 \\
\hline $\mathrm{PCO}_{2}$ pre-VAFO $(\mathrm{mmHg})$ & $59 \pm 17,8$ & $60,96 \pm 19,38$ & 0,75 \\
\hline Puntaje de Murray & $2,79 \pm 0,68$ & $3,23 \pm 0,58$ & 0,003 \\
\hline VMC pre-VAFO (días) & $6,1 \pm 10$ & $10,28 \pm 19$ & 0,27 \\
\hline PELOD al ingreso a VAFO (\%) & $7,19 \pm 8,7$ & $20,71 \pm 22,3$ & 0,0009 \\
\hline Paw máxima en VAFO (mmHg) & $26,9 \pm 4,97$ & $32,23 \pm 5,5$ & 0,002 \\
\hline $\mathrm{FiO} 2>0,6 \times 24 \mathrm{~h}(\mathrm{n}=38)$ & $26,3 \%(n=10)$ & $73,7(n=28)$ & 0,0001 \\
\hline Adrenalina $>0,25 \mu / \mathrm{kg} / \min (\mathrm{n}=33)$ & $24,2 \%(n=8)$ & $75,8 \%(n=25)$ & 0,001 \\
\hline Hemofiltración $(n=8)$ & $12,5 \%(n=1)$ & $87,5 \%(n=7)$ & 0,073 \\
\hline
\end{tabular}

PIM2: índice de mortalidad pediátrica; VMC: ventilación mecánica convencional; VAFO: ventilación oscilatoria de alta frecuencia; Paw: presión media de la vía aérea; IO: índice de oxigenación; PAFI: $\mathrm{PaO}_{2} / \mathrm{FIO}_{2}$; PELOD: puntaje de disfunción multiorgánica (Pediatric Logistic Organ Dysfunction). 
minar si la utilización de uno u otro aportaba beneficio alguno. A excepción de la edad y el peso, ambos inferiores en el grupo tratado con SLE $5000(12 \pm 17$ meses y $7,2 \pm 4,68 \mathrm{~kg}$ contra $52 \pm 55$ meses y $20,28 \pm 13,8 \mathrm{~kg}$ ), no se encontraron diferencias significativas ni en los parámetros de oxigenación, ni en la duración de la VMC previo a VAFO, como así tampoco en la mortalidad.

\section{DISCUSIÓN}

La ARM es un tratamiento indispensable para disminuir la mortalidad del SDRA, pero también puede ser un factor agravante en sí mismo y las estrategias ventilatorias son todavía motivo de controversia. En ese contexto, la VAFO es un recurso que fisiopatológicamente parece ideal, pero sin que sus logros clínicos hayan sido definitivamente probados. Sin embargo, el aumento de la evidencia generada, lograda en niños y adultos, parece tender a demostrar su beneficio, como lo muestra un metaanálisis publicado a mediados del 2010 sobre el uso de la ventilación de alta frecuencia oscilatoria en niños y adultos, que halló que disminuía la mortalidad en alrededor de un $25 \%(p=0,03)$ y en un $35 \%$ la ocurrencia de hipoxemia refractaria, hipercapnia, hipotensión, o barotrauma $(p=0,04) .{ }^{16}$ Este estudio fue evaluado y publicado en los Resúmenes de Revisión Sistemática con Calidad Evaluada de la Colaboración Cochrane, quienes concluyen que la Revisión Sistemática está bien realizada, que las conclusiones son prudentes, basadas en los datos y confiables. ${ }^{19}$ En ese contexto es importante la evaluación de los resultados en el uso rutinario de esta tecnología. Por ello, dentro de las restricciones que plantea el diseño retrospectivo, este estudio permite una evaluación de la efectividad de esta técnica ventilatoria en la práctica cotidiana de un centro terciario de referencia que no dispone de ECMO como recurso terapéutico. En tal análisis se lo debe diferenciar de los estudios de eficacia que analizan los resultados en situaciones ideales/experimentales. ${ }^{20}$ En ese sentido, de las diversas series publicadas, ${ }^{21-33}$ el análisis de efectividad más numeroso y detallado publicado es el de Arnold. ${ }^{34}$ Se trata de un detallado estudio retrospectivo multicéntrico de 10 hospitales pediátricos terciarios universitarios de EE.UU. y Canadá. Este trabajo informa 232 pacientes, de los cuales el 49,6\% necesitaron ECMO o fallecieron antes de los 28 días. Asimismo, el $11 \%$ de sus pacientes fueron inmunocomprometidos y el análisis multivariado halló que tales pacientes tenían 5 veces más probabilidad de morir que los pacientes no-inmunocomprometidos. Nuestra serie con un $34 \%$ de inmunocomprometidos, un $200 \%$ más frecuente que en la serie de Arnold y una mortalidad de $73 \%$ en tales pacientes avala la asociación de inmunocompromiso y mortalidad en el SDRA grave. En forma similar, los pacientes comunicados por Slee-Wijffels, con una mortalidad global de $35,8 \%$, tenían una mediana de PIM2 de 2,8\% en sobrevivientes y 3,9\% en no sobrevivientes, ${ }^{35}$ mientras que nuestra serie tuvo $2,3 \%$ y $14 \%$, respectivamente.

En otro trabajo retrospectivo, Brogan estudió una serie de 66 pacientes con una mortalidad global de $60 \%$ y mostró que la mortalidad crece de acuerdo al número creciente de fallos de órganos, desde $12 \%$ con un fallo, $67 \%$ con 2 fallos y hasta $95 \%$ de mortalidad en los pacientes con 3 o más fallos de órganos. ${ }^{21}$ También en nuestros pacientes pudimos observar una significativa diferencia, entre sobrevivientes y fallecidos, en el número de fallos de órganos al ingreso a VAFO, medidos a través del puntaje PELOD.

La mayoría de los factores asociados a mortalidad hallados en este estudio, tales como las condiciones clínicas de ingreso, enfermedad crónica subyacente y mortalidad predicha por puntajes estandarizados, no son manipulables terapéuticamente por el médico y probablemente tampoco lo sean los factores que muestran mala evolución, como incapacidad de disminuir primero la $\mathrm{FIO}_{2}$ y luego la $\mathrm{Paw}^{34}$ requerimientos ascendentes de soporte cardiovascular o progresión del fallo de otros órganos. Sin embargo, estos datos imponen la búsqueda de recursos pronósticos más sofisticados que le ahorre al paciente y su familia pruebas terapéuticas que solo postergan el óbito y que, a su vez, permitan un uso rutinario de esta tecnología más efectiva. Esto es tanto más importante cuanto menor sea la disponibilidad de VAFO en un hospital o región dada.

Respecto de la oportunidad de ingreso a VA$\mathrm{FO}$, si bien el IO de ingreso a VAFO se encontraba por encima del sugerido por la bibliografía $(25,6 \pm 13)$, éste era similar al descrito por varias publicaciones y la experiencia multicéntrica ya citada, con un IO medio de 27,1 al inicio de la VAFO. ${ }^{34}$ Sin embargo, el hecho de que al ingreso a VAFO los pacientes fallecidos tenían un IO más alto, una PAFI más baja y un puntaje de Murray con mayor compromiso pulmonar, junto con la tendencia, aunque no significativa, de haber esta- 
do más tiempo en ARMc (10 contra 6 días) antes de recibir VAFO, abre el interrogante, tal como lo sugieren otros trabajos, ${ }^{23,35}$ de si un ingreso más temprano no hubiera disminuido la mortalidad.

\section{CONCLUSIÓN}

En una población con alta prevalencia de enfermos crónicos, oncológicos e inmunocomprometidos, el uso rutinario de la VAFO se asoció con un 33,4\% de supervivencia. Se necesitan más estudios pronósticos que permitan una selección más efctiva de los pacientes candidatos a esta tecnología.

\section{Agradecimiento}

Al Lic. Juan Pablo Bonora por su colaboración desinteresada en la recolección de datos.

\section{BIBLIOGRAFÍA}

1. Arnold JH, Ventre KM. Acute Lung Injury and Acute Respiratory Distress Syndrome. En: Nichols DG, (ed). Roger's Textbook of Pediatric Intensive Care. $4^{\text {th }}$ ed. Filadelfia: Lippincott Williams \& Wilkins; 2008.Págs.731-52.

2. Selandari JO. Ventilación de alta frecuencia y síndrome de dificultad respiratoria aguda. Med Infantil 1998;5(1):51-6.

3. Selandari JO, Landry L. Síndrome de distress respiratorio agudo. Criterios de atención en UCIP. Buenos Aires: Fundación Hospital de Pediatría “Prof. Dr. Juan P. Garrahan”; 2002.

4. Landry L, Motto E. Síndrome de dificultad respiratoria aguda. En: Vassallo JC, Rufach D, (eds.). Manual de Emergencias y Cuidados Críticos en Pediatría. Buenos Aires: FUNDASAP; 2009.Págs.107-12.

5. MacIntyre NR. Current issues in mechanical ventilation for respiratory failure. Chest 2005;128(5 Suppl 2):561S-7S.

6. Villar J. Ventilator or physician-induced lung injury? Minerva Anestesiol 2005;71(6):255-8.

7. Lionetti V, Recchia FA, Ranieri VM. Overview of ventilator-induced lung injury mechanisms. Curr Opin Crit Care 2005;11(1):82-6.

8. Gajic O, Dara SI, Méndez JL, Adesanya AO, et al. Ventilator-associated lung injury in patients without acute lung injury at the onset of mechanical ventilation. Crit Care Med 2004;32(9):1817-24.

9. Webb HH, Tierney DF. Experimental pulmonary edema due to intermittent positive pressure ventilation with high inflation pressures. Protection by positive end-expiratory pressure. Am Rev Respir Dis 1974;110(5):556-65.

10. Dreyfuss D, Soler P, Basset G, Saumon G. High inflation pressure pulmonary edema. Respective effects of high airway pressure, high tidal volume, and positive end-expiratory pressure. Am Rev Respir Dis 1988;137(5):1159-64.

11. Gattinoni L, Pesenti A, Avalli L, Rossi F, Bombino M. Pressure-volume curve of total respiratory system in acute respiratory failure. Computed tomographic scan study. Am Rev Respir Dis 1987;136(3):730-6.

12. ARDSnet Study Group. Ventilation with lower tidal volumes as compared with traditional tidal volumes for acute lung injury and the acute respiratory distress syndrome. The Acute Respiratory Distress Syndrome Network. N Engl J Med 2000;342:1301-8.
13. Arnold JH, Truog RD, Thompson JE, Fackler JC. Highfrequency oscillatory ventilation in pediatric respiratory failure. Crit Care Med 1993;21(2):272-8.

14. García MD, Selandari JO. Ventilación de alta frecuencia. En: Vassallo JC, Rufach D, (eds). Manual de Emergencias y Cuidados Críticos en Pediatría. Buenos Aires: FUNDASAP; 2009.Págs.113-20.

15. Arnold JH, Hanson JH, Toro-Figuero LO, Gutiérrez J, et al. Prospective, randomized comparison of high-frequency oscillatory ventilation and conventional mechanical ventilation in pediatric respiratory failure. Crit Care Med 1994;22(10):1530-9.

16. Sud S, Sud M, Friedrich JO, Meade MO, et al. High frequency oscillation in patients with acute lung injury and acute respiratory distress syndrome (ARDS): systematic review and meta-analysis. BMJ 2010;340:c2327.

17. Leteurtre S, Martinot A, Duhamel A, Proulx F, et al. Validation of the paediatric logistic organ dysfunction (PELOD) score: prospective, observational, multicentre study. Lancet 2003;362(9379):192-7.

18. Murray JF, Matthay MA, Luce JM, Flick MR. An expanded definition of the adult respiratory distress syndrome. Am Rev Respir Dis 1988;138(3):720-3.

19. NHSCentre for Reviews and Dissemination databasenumber: DARE12010003427:SudS, Sud M,Friedrich JO, Meade MO, Ferguson ND, Wunsch H, et al. High frequency oscillation in patients with acute lung injury and acute respiratory distress syndrome (ARDS): systematic review and meta-analysis (Structured abstract). BMJ 2010;340:c2327. [Acceso: 29 de mayo de 2011]. Disponible en: http:/ / cochrane.bvsalud.org/cochrane/show.php?db=dare_abstra cts\&mfn=5037\&id=DARE12010003427\&lang=es\&dblang $=\& l i b=$ COC \&print $=$ yes

20. González De Dios J. De la medicina basada en la evidencia a la evidencia basada en la medicina. An Esp Pediatr 2001;55(5):429-39.

21. Brogan TV, Bratton SL, Meyer RJ, O'Rourke PP, Jardine DS. Nonpulmonary organ failure and outcome in children treated with high-frequency oscillatory ventilation. J Crit Care 2000;15(1):5-11.

22. Duval EL, Markhorst DG, Gemke RJ, van Vught AJ. Highfrequency oscillatory ventilation in pediatric patients. Neth J Med 2000;56(5):177-85.

23. Fedora M, Klimovic M, Seda M, Dominik P, Nekvasil R. Effect of early intervention of high-frequency oscillatory ventilation on the outcome in pediatric acute respiratory distress syndrome. Bratisl Lek Listy 2000;101(1):8-13.

24. Martinón TF, Rodríguez NA, Jaimovich DG, Martinón Sánchez JM. High-frequency oscillatory ventilation in pediatric patients. protocol and preliminary results. An Esp Pediatr 2000;53(4):305-13.

25. Pillow JJ, Wilkinson MH, Neil HL, Ramsden CA. In vitro performance characteristics of high-frequency oscillatory ventilators. Am J Respir Crit Care Med 2001;164(6):1019-24.

26. Selandari JO, Vassallo JC, Collman C, Torres S, Sasbón JS. Ventilación de alta frecuencia oscilatoria en pediatría. Arch Argent Pediatr 2001;99(5):397-404.

27. Donoso AF, León JB, Rojas G, Valverde CG, et al. Uso de ventilación de alta frecuencia oscilatoria en pacientes pediátricos. Rev Chilena Pediatr 2002;73(5):461-70.

28. Ben JN, Khaldi A, Mnif K, Bouziri A, et al. High-frequency oscillatory ventilation in pediatric patients with acute respiratory failure. Pediatr Crit Care Med 2006;7(4):362-7.

29. García Hernández JA, Vázquez FA, Martínez López AI, González Rodríguez JD, et al. High-frequency oscilla- 
tory ventilation. A single-center study. An Pediatr (Barc) 2006;65(1):67-72.

30. Cruces Romero P, Donoso Fuentes A, León Bravo J, Valenzuela Vásquez J, Camacho Álvarez J. Ventilación de alta frecuencia oscilatoria en el rescate de hipoxemia catastrófica en niños con síndrome de distrés respiratorio agudo. Rev Chil Med Intensiv 2007;22(1):7-14.

31. Wang CC, Wu WL, WuET, Chou HC, Lu FL. High frequency oscillatory ventilation in children: experience of a medical center in Taiwan. J Formos Med Assoc 2008;107(4):311-5.

32. Yánez P, Lapadula A, Benavente R, Von DG, Emilfork SM.
Ventilación de alta frecuencia en infección respiratoria grave por VRS. Rev Chilena Pediatr 2010;81(3):221-7.

33. Rodríguez C, Ronco M, Castillo M, Guzmán R, et al. Ventilación oscilatoria de alta frecuencia en niños con síndrome de dificuldad respiratoria del adulto. Arch Argent Pediatr 2011;98(3):175-81.

34. Arnold JH. High-frequency ventilation in the pediatric intensive care unit. Pediatr Crit Care Med 2000;1(2):93-9.

35. Slee-WijffelsFY, VanD, V, TwiskJW, MarkhorstDG, Plotz FB. High-frequency oscillatory ventilation in children: a singlecenter experience of 53 cases. Crit Care 2005;9(3):R274-R279.

¿Qué es la sensatez? Podríamos definirla como una sabiduría práctica: hacer bien lo que hay que hacer, y no hacer -ni bien ni mal-lo que no hay que hacer. Hasta hace apenas décadas las insensateces eran resultado de la ignorancia y las creencias; en los últimos tiempos, el conocimiento científico ha ido reduciendo las insensateces de la ignorancia, y sin embargo, no ha sido tan exitoso en reducir las de las creencias.

Alberto Agrest 